\section{Analysis of an Integrated Lens Antenna Fed by SIW Slot Array Using a Hybrid MoM-PO Method}

\author{
R. Bayderkhani, K. Forooraghi, Member, IEEE, E. Arnieri, \\ Member, IEEE, B. Abbasi-Arand, Member, IEEE, and B. \\ Virdee, Member IEEE
}

\begin{abstract}
This paper presents a very fast and highly efficient full-wave hybrid method for analyzing an integrated dielectric lens antenna (ILA) fed by multilayered substrate integrated waveguide (SIW) slot antenna/array. The feeding antenna structure is modeled as a stacked parallel-plate waveguide with metallic posts, coupling and radiating slots. Physical optics (PO) method in conjunction with 3D ray tracing technique is employed to analyze the effect of the dielectric lens on the SIW feeding slots. Fields in the SIW structure are computed by considering the Dyadic Green's function expressed as an expansion of vectorial cylindrical eigenfunctions and taking into account scattering at the conducting posts. Slots are modeled with equivalent magnetic currents expressed as a sum of domain basis functions. By imposing continuity of the tangential components of the fields an integral equation is obtained that is solved with the application of Method-of-Moments (MoM). In order to validate the proposed technique, a hemispherical ILA fed by a double-layered SIW cavity which is backed with slot antenna is analyzed. Excellent agreement is obtained with HFSS software together with significant improvement in computational time and memory requirements.
\end{abstract}

Index Terms - Dyadic Green's function, hybrid methods, integrated lens antenna, method-of-moments, ray/physical optics technique, slot antenna, substrate integrated waveguide.

\section{INTRODUCTION}

INTEGRATED dielectric lens antennas (ILA), also referred to as substrate lens, are widely used in various communication, radar, and imaging systems, especially in the millimeter ( $\mathrm{mm})$ and sub-mm wave bands [1]. This is because the major drawbacks of traditional dielectric lens and planar antennas, such as low directivity and losses of power into surface waves, can be overcome when a dielectric lens is leaned against the planar antenna, the latter providing the primary focal source for the lens itself. This makes ILAs compatible with the integration in RF front-end circuits. Microstrip patch antennas/arrays commonly used in ILAs [1]-[4] however are unsuitable for application at the millimeter wave band because they possess higher ohmic losses in these high frequencies.

Over the last few decades, substrate integrated waveguide (SIW) technology has been widely used in the design of

Manuscript received February 19, 2015.

R. Bayderkhani, K. Forooraghi and B. Abbasi-Arand are with the Department of Electrical and Computer Engineering, University of Tarbiat Modares (TMU), Jalal-Al-Ahmad Highway, Tehran, IRAN (e-mail: keyvan_f@modares.ac.ir).

E. Arnieri is with the Department of Informatics, Modeling, Electronics and System Engineering (DIMES), University of Calabria-87036 Rende (CS), ITALY

B. Virdee is Director of Center for Communications Technology, London Metropolitan University, London, UK numerous structures and antennas from microwave to sub-mm wave bands [5],[6]. The use of SIW-based slot antenna/array to feed dielectric lens in ILAs is becoming very attractive at these frequency bands.

There are many instances in recent literature discussing the methods for analyzing ILAs using various numerical approaches commonly based on geometrical optics (GO), physical optics (PO) principles [2]-[4], and FDTD method [7],[8]. Moreover, SIW circuits have been designed and analyzed using approximate analytical methods based on transmission-line theory [9], the equivalent-waveguide-width model [10], two dimensional, multi-port methods [11] or finite-element (FEM) and finite-difference (FD) based commercial full wave solvers. The former approach is computationally efficient, but it does not guarantee the accuracy of the final design results. The latter approach can be very time consuming and memory exhaustive especially for large and complex structures. Hence, an efficient full-wave analysis technique is required in order to investigate general ILA structures fed using SIW slot antenna/array.

In this paper, a very fast and highly efficient full-wave technique is proposed for analyzing an ILA structure fed by SIW-based slot antenna/array. The approach in [12] has been extended to analyze a double-layered SIW slot antenna/array with arbitrary number of radiating and/or coupling slots. In addition, to predict the effects of dielectric lens on the SIW antenna, the dielectric lens is analyzed using Ray/PO technique. Finally, the equations with the unknowns are solved to obtain the equivalent surface currents on the aperture of slots. The proposed technique is validated by applying it to a hemispherical ILA fed by a double layered SIW-based cavity backed slot antenna. Comparison between the proposed technique and finite element method using HFSS are presented, which show the proposed technique is exceptionally efficient in terms of computational time and storage capacity, as well as numerical accuracy.

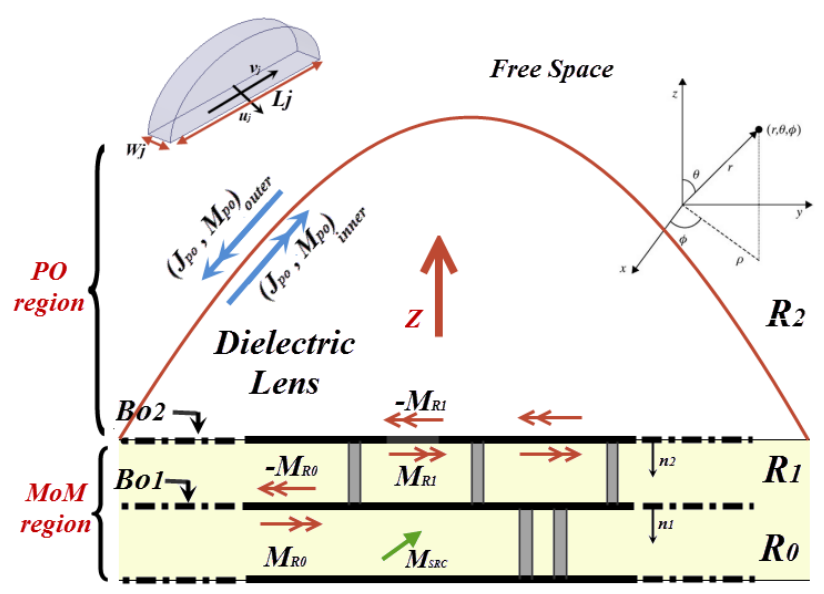

Fig.1 Schematic view of an ILA fed by double layered SIW slot antenna. Also shown is an entire domain basis function shape used to expand the unknown magnetic current on the slot with corresponding parameters.

II. PROBLEM FORMULATION

The solution domain is split into three distinct regions, as 
shown in Fig. 1, which are connected by the unknown magnetic currents $M$. MoM regions $R 0$ and $R 1$ are include a generic SIW circuit with arbitrary number of coupling/radiating slots and posts excited through coaxial/waveguide ports. $R 2$ is the $\mathrm{PO}$ region away from the antenna containing a dielectric lens which is stacked on the top wall of the antenna and characterized by the constitutive parameters $\varepsilon_{d}$ and $\mu_{d}$. Bor denotes the boundary surface between $R 0$ and $R l$, and $B o_{l}$ is the boundary between $R 0$ and $R 1$. The coupling slots are located on $B o l$, while the radiating slots are placed on Boz. The normal vector $\hat{n}_{i+1}$ points into the region Ri. Parallel plates that form the SIW structure are assumed to be a perfect-electric conductor with infinitesimal thickness. Only one excitation is considered here however if several feeding ports are present their effects would be superposed. Excitation port generates the field in Ro and the same time the field in $R l$ is excited by the coupling slots placed on Bor.

In order to model the double-layered SIW-based slot antenna (internal region) MoM along with mode matching technique are necessary. This is done by representing the field in the post walled structure through the Dyadic Green's function of the parallel plate waveguide [13],[14]. By invoking the field-equivalence principle the slots are replaced by a metal plate and equivalent magnetic currents $\left(M_{\text {inner }}\right.$ and $\left.M_{\text {outer }}\right)$ are introduced on their respective surfaces. Due to the use of narrow rectangular slots it can be assumed that there are only transverse electric field components across the slots, and the magnetic current is completely directed along the longitudinal direction and its transverse dependence is negligible. Applying the boundary conditions on the aperture of slots requires the tangential electric and magnetic fields to be continuous. The continuity of tangential electric fields leads to the equality $M_{\text {inner }}=-M_{\text {outer }}=M$, as shown in Fig. 1 , whereas the continuity of magnetic field across the surface $S$ of the slots leads to the following general integral equation for each slot $i$ :

$$
\begin{aligned}
& \hat{n} \times H_{\text {tot }}^{\text {inner }}(r)=\hat{n} \times H_{\text {tot }}^{\text {outer }}(r) \\
& \hat{n} \times\left(H_{\text {inc }}^{\text {inner }}(r)+\sum_{j}^{N s} H_{M_{-} \text {slot }}^{\text {inner }}(r)\right)= \\
& =\hat{n} \times\left(\begin{array}{l}
H_{\text {inc }}^{\text {outer }}(r)+ \\
+\sum_{j}^{N s} H_{M_{-} \text {slot }}^{\text {outer }}(r)+\sum_{q}^{N q} \sum_{j}^{N s} H_{j q}^{P o}(r)
\end{array}\right) \quad r \in S_{i}
\end{aligned}
$$

In which [12]

$$
\begin{aligned}
& H_{i n c}(r)=-j \omega \varepsilon_{0} \varepsilon_{r} \iint_{V_{S R C}} \bar{G}_{P P W}\left(r, r^{\prime}\right) \cdot M_{S R C}\left(r^{\prime}\right) d r^{\prime}+H_{S}^{M_{S R C}}(r) \\
& H_{M_{-} s l o t, j}(r)=-j \omega \varepsilon_{0} \varepsilon_{r} \iint_{S_{j}} \bar{G}_{P P W}\left(r, r^{\prime}\right) \cdot M_{j}\left(r^{\prime}\right) d r^{\prime}+H_{S}^{M_{j}}(r)
\end{aligned}
$$

Where $r$ and $r^{\prime}$ define the observation and source point, respectively; $N_{s}$ is the number of slots; $N q$ is the number of modes used to expand the current on the slot.

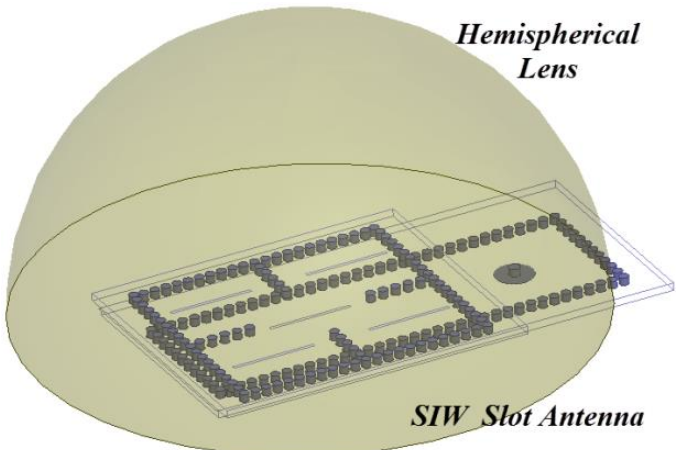

Fig.2 Representation of the entire hemispherical lens antenna.

$M_{j}$ is the equivalent magnetic current source defined on surface of slot $j\left(\mathrm{~S}_{\mathrm{j}}\right) ; \bar{G}_{P P W}\left(r, r^{\prime}\right)$ is the Dyadic Green's function of the parallel plate waveguide when all the slots are absent (see Appendix A); $H_{S}^{M}$ is the field scattered by the metallic posts due to the current $M ; \mathrm{M}_{S R C}\left(r^{\prime}\right)$ is the magnetic current source distribution defining the port excitation in volume $V_{S R C}$ located in region $R_{0}$; and $H^{P O}$ is the magnetic field pre-computed by Ray/PO technique to model the dielectric lens effects, and it has non zero value only for outer region. To do so, the equivalence principle is applied on the lens surface and the forward as well as backward radiation is calculated. The total field in the external region is then obtained by the summation of incident fields impinging from the antenna and scattered fields from the lens boundary. Surface integration equation was established on the aperture of slots. Finally, the equations with the unknowns are solved to obtain the equivalent surface currents on the aperture of slots. In the application of (2), it should be noted that in each region only corresponding slots should be considered, for example in $R o$ the radiating slots are vanished.

The equivalent magnetic currents, which are used to model the slots, are expanded in terms of an entire domain sinusoidal basis function. The magnetic currents for a slot $j$ are:

$$
\begin{aligned}
& M_{j}\left(r^{\prime}\right)=\hat{v}_{j} \sum_{q} f_{q, j}\left(r^{\prime}\right) . V_{q, j} \quad 1 \leq j \leq N_{s} \\
& f_{q, j}\left(r^{\prime}\right)=\sin \left(k_{q}\left(\frac{L_{j}}{2}+v_{j}\right)\right), \quad\left|v_{j}\right| \leq L_{j} \\
& k_{q}=\frac{q \pi}{L_{j}} \quad \text { and } \quad r^{\prime}=r_{j q}+u \hat{u}_{j}+v \hat{v}_{j}
\end{aligned}
$$

Where $r_{j q}, V_{q, j}$ and $L_{j}$ are defined as the center of the $q$ th basis function on the slot $j$, the unknown expansion coefficients and the length of the jth slot, respectively, as shown in Fig. 1, and $q$ spans over the number of modes $N q$.

The integral equation (2) is solved by using Galerkin weighting procedure where equations (3)-(7) are substituted into the (2). After some mathematical manipulations, the following linear system with $N_{s} \times N_{q}$ unknowns is obtained:

$$
h_{p, i}=\sum_{q, j} Y_{q, j}^{p, i} \cdot V_{q, j} \quad \forall(p, i)
$$


Where $p$ and $q$ span over the basis functions, while $i$ and $j$ span, the number of slots, $N S$.

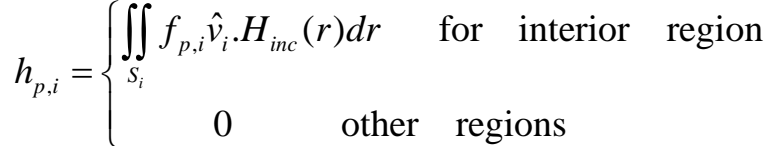

$$
\begin{aligned}
& Y_{q, j}^{p, i}=Y_{q, j}^{p, i}(\text { int } 1)+Y_{q, j}^{p, i}(\text { int } 2)+Y_{q, j}^{p, i}(e x t)
\end{aligned}
$$

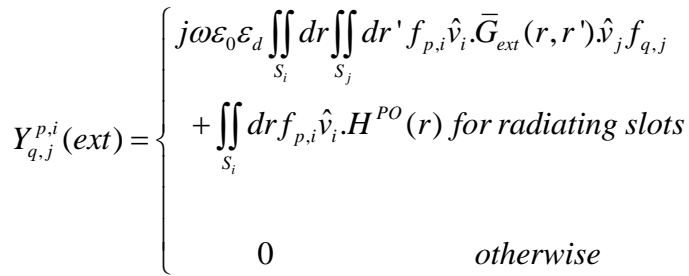

$$
\begin{aligned}
Y_{q, j}^{p, i}(\mathrm{int})= & j \omega \varepsilon_{0} \varepsilon_{r} . \\
& \iint_{S_{i}} d r \iint_{S_{j}} d r^{\prime} f_{p, i} \hat{v}_{i} \cdot \bar{G}_{P P W}\left(r, r^{\prime}\right) \cdot \hat{v}_{j} f_{q, j} \\
& +\iint_{S_{i}} d r f_{p, i} \hat{v}_{i} \cdot H_{S}^{f_{q, j}}(r)
\end{aligned}
$$

Where $Y_{q, j}^{p, i}($ int 1$)$ and $Y_{q, j}^{p, i}($ int 2$)$ are the admittance elements in $R 0$ and $R 1$ regions, respectively, that have the same form of (12). $G_{e x t}$ is the Dyadic Green's function for the external region [12]. The incident magnetic field radiating into the structure by a port source in the absence of the slots is defined by $H_{\text {inc }}(r)$. In the above formulation only one port source which is radiated in interior region is assumed. As mentioned earlier, if several feeding ports are presented, their effects need to be superposed. Consequently, the number of unknowns is given by the number of modes assumed in each slot $(\mathrm{Nq})$ multiplied by the number of slots $\left(N_{S}\right)$, i.e. $N q \times N s$. As a result, the computational efficiency is achieved along with reasonable accuracy by using the proposed hybrid method.

\section{Simulation}

In order to validate the proposed technique it was applied to a hemispherical ILA fed by a double layered SIW based cavity backed slot antenna. A comparison between the proposed method and finite element simulations carried out using Ansoft's HFSS are presented and discussed.

\section{A. Input Impedance and Radiation Pattern}

The geometry of the hemispherical ILA fed by double layered SIW cavity backed slot antenna is shown in Fig.2, and the corresponding parameters shown in Fig.3. The SIW antenna structure consists of two main building blocks consisting of the feeding system and the radiating system, as shown in Fig.3. The feeding system and the radiating system are implemented on two different substrate layers. The vertical walls of structure are realized using rows of metalized posts embedded in a dielectric substrate (RO4003TM) with a relative permittivity of 3.55, a loss tangent of 0.0027 and a thickness of $10 \mathrm{mil}$. The radiating system includes a main cavity, where the radiating slots are etched on the top side metal, as shown in Fig.2.

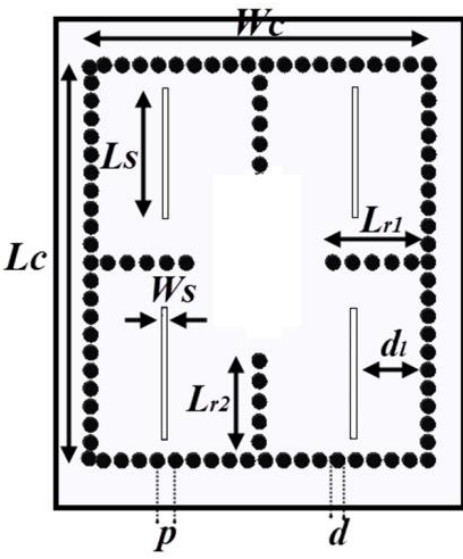

(a)

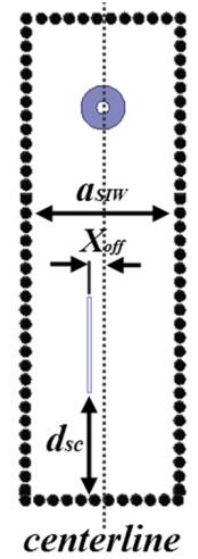

(b)
Fig.3 Feeding SIW slot antenna including: (a) cavity and radiating slots placed on top of the substrate, and (b) feeding waveguide and coupling aperture placed on bottom of the substrate.

TABLE I

ANTENNA PARAMETERS AND CORRESPONDING VALUES

\begin{tabular}{c|c|c|c}
\hline \hline Parameter & Value $(\mathrm{mm})$ & Parameter & Value $(\mathrm{mm})$ \\
\hline \hline $\mathrm{Lc}$ & 9.09 & $\mathrm{p}$ & 0.43 \\
\hline $\mathrm{Wc}$ & 7.77 & $\mathrm{~d}$ & 0.35 \\
\hline $\mathrm{Ls}$ & 3 & $\mathrm{Lr} 1$ & 2.02 \\
\hline $\mathrm{Ws}$ & 0.117 & $\mathrm{Lr} 2$ & 2.29 \\
\hline $\mathrm{d} \mathrm{l}$ & 1.63 & $\mathrm{dsc}$ & 3.37 \\
\hline $\mathrm{a}_{\text {SIw }}$ & 4.8 & Xoff & 0.423 \\
\hline \hline
\end{tabular}

The cavity is divided into four sub-sections with a radiating slot on the top wall of each sub-section. In order to excite the cavity, a feeding short circuited SIW waveguide on the rear side of the cavity is used. In order to connect two layers a coupling longitudinal slot transition located in common face of two SIW blocks is used. This antenna radiates into a hemispherical dielectric lens with constitutive parameters $\varepsilon_{d}=2.1$ and $\mu_{d}=1$ and radius of $\mathrm{R}_{\mathrm{DL}}=50 \mathrm{~mm}$. The antenna parameters and their corresponding values are listed in Table I. Fig.4(a) shows the reflection coefficient of the antenna compared with results obtained from HFSS. The phase of the reflection coefficient of the antenna is depicted in Fig.4(b). The total field pattern of the antenna is shown in Fig.5. The results indicate that the proposed method agrees very well with the results using HFSS. It should be noted that by tuning the ILA parameters, better matching is achievable. In order to show the dielectric lens effects on the input impedance of the antenna, the impedance with and without dielectric lens is computed and shown in Fig.6; from this figure, it is found that the input impedance of the antenna is strongly altered due to the presence of lens.

\section{B. CPU Time and Storage Requirements}

The hybrid method described in the present paper requires three matrices to be filled and inverted. In the PO region, a matrix needs to be filled in order to compute the PO currents on the lens surface. 


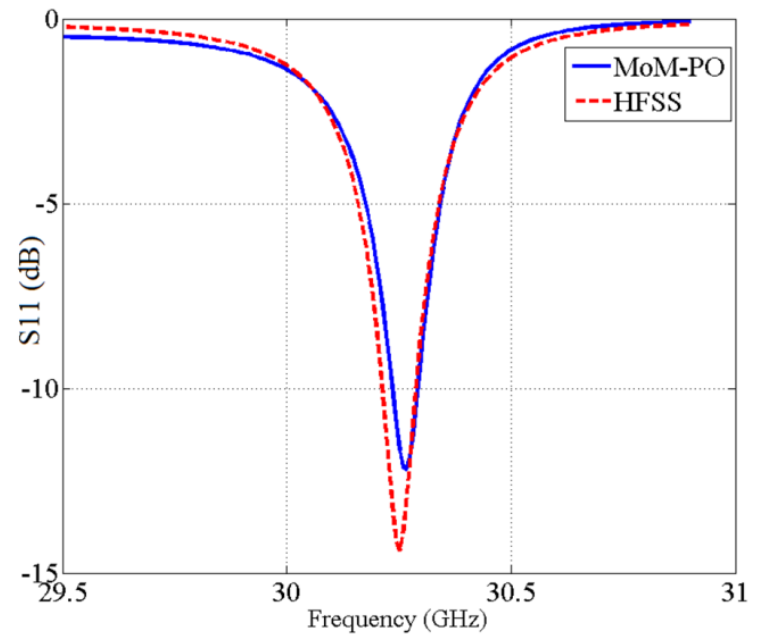

(a)

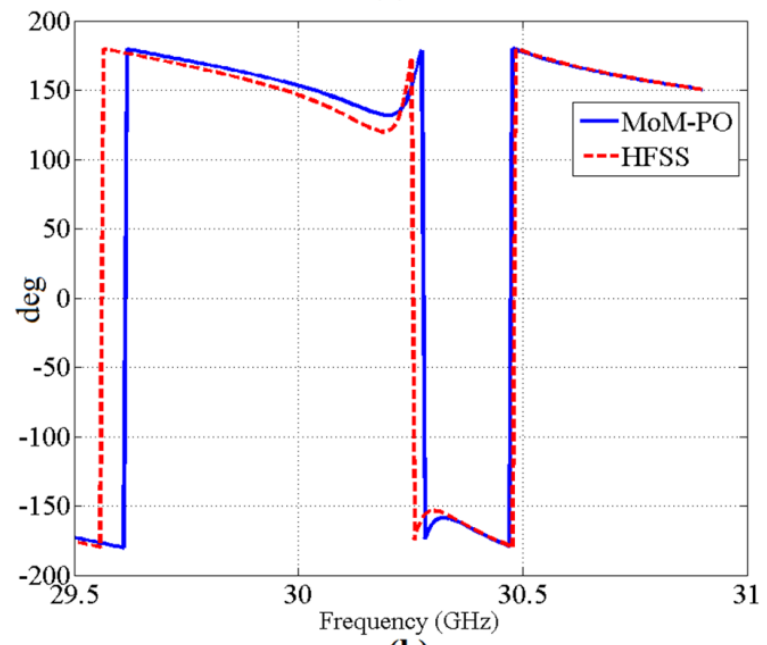

(b)

Fig.4 Reflection coefficient of the ILA compared with HFSS, (a) magnitude, and (b) phase.

The matrix dimension depends on the number of points assumed in order to compute the PO currents. In the MoM region, two matrixes need to be filled, where one of them used to compute the field scattered by the metallic vias and the other one to evaluate the unknown magnetic currents over the slots.

The size of matrix in PO region depends on the area that is illuminated by the antenna beam, and the number of mesh cells depends on the computational area. By increasing the number of mesh cells the accuracy of the PO fields and consequently the $\mathrm{CPU}$ processing time and memory requirements are increased. The simulated results indicate that a $\lambda_{g} / 10 \times \lambda_{g} / 10$ mesh cell size covering the illuminated area is sufficient to ensure the accuracy of the calculated PO fields. In MoM region, the number of cylinders $N C$, the number of slots $N s$ and the number of $Z$ modes $\mathrm{Nz}$ define the size of two matrixes.

Table II and III report the CPU processing time and memory storage used in simulating the hemispherical ILA fed by the double layered SIW cavity backed slot antenna, respectively.
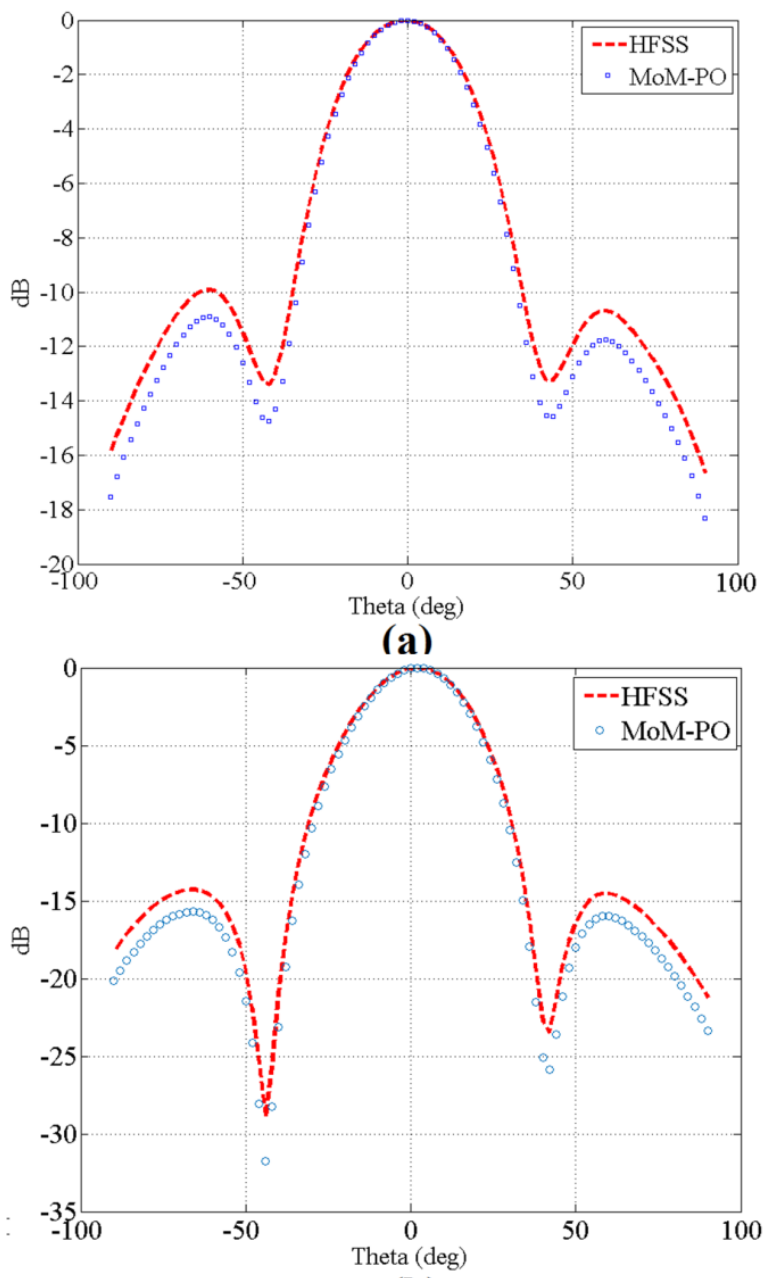

(b)

Fig.5 Total field pattern of the ILA computed using the proposed technique and HFSS in (a) $\varphi=0$ plane, and (b) $\varphi=90$ plane.

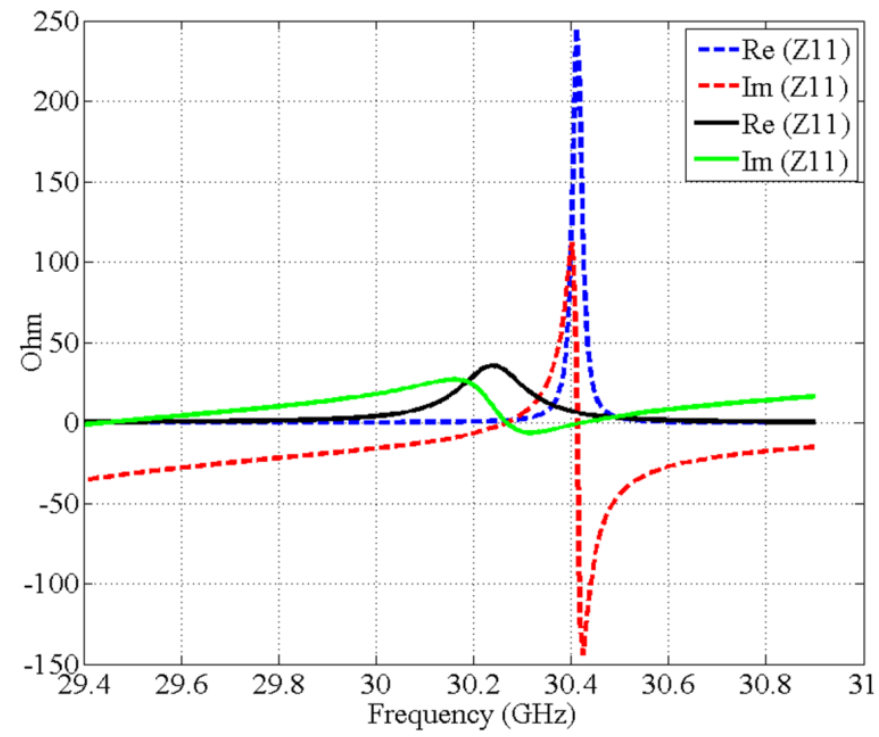

Fig.6 Impact of dielectric lens on the input impedance of the SIW slot antenna obtained by using MoM-PO method (solid-lines show the Z11 with lens effects and dash-lines show Z11 without lens effects). 
TABLe II

CPU TIME (INTEL CORE I7 3.55 GHZ, 16 GB RAM)

\begin{tabular}{c|c|c|c}
\hline \hline \multirow{2}{*}{ Structure type } & \multicolumn{2}{|c|}{ HFSS - CPU time } & This paper \\
\cline { 2 - 4 } & Mesh setup & Freq. point & Freq. point \\
\hline Antenna without lens & $74 \mathrm{~s}$ & $65 \mathrm{~s}$ & $3 \mathrm{~s}$ \\
\hline Antenna with lens & $128 \mathrm{~s}$ & $101 \mathrm{~s}$ & $5.6 \mathrm{~s}$ \\
\hline \hline
\end{tabular}

TABLE III

MEMORY REQUIREMENT

\begin{tabular}{c|c|c}
\multicolumn{3}{|c}{ MEMORY REQUIREMENT } \\
\hline \hline Structure type & HFSS & This paper \\
\hline Antenna without lens & $3.01 \mathrm{~GB}$ & $0.12 \mathrm{~GB}$ \\
\hline Antenna with lens & $8.33 \mathrm{~GB}$ & $0.22 \mathrm{~GB}$ \\
\hline \hline
\end{tabular}

Comparison of the CPU time and storage capacity of the proposed technique with HFSS shows substantial reduction is achieved with the proposed technique, i.e. CPU time is reduced by around $95 \%$ and memory capacity is reduced by around $96 \%$. Consequently, the proposed technique raises possibility of simulating larger and more complex structures such as multilayered SIW-based antennas with limited computing resources with acceptable numerical accuracy.

\section{CONCLUSION}

A very fast and highly efficient full-wave technique is proposed that is based on a hybrid MoM-PO implementation that enables rigorous analyzes of ILAs fed by SIW-based slot antenna/array. The technique was applied to analyze a hemispherical ILA fed by double-layered SIW cavity backed slot antenna. Excellent agreement is obtained between the proposed technique and commercial FEM-based solver like HFSS. However the proposed technique offers the advantage of providing a solution at a significantly shorter computational time frame with substantially lower storage requirements without compromising numerical accuracy.

\section{APPENDIX A}

The Dyadic Green's function of the parallel plate waveguide has the following form [13]:

$$
\begin{aligned}
& \bar{G}_{P P W}\left(r, r^{\prime}\right) \\
&=-\frac{1}{k^{2}} \hat{z} \hat{z} \delta\left(r-r^{\prime}\right)-j \sum_{m}\left(1-\frac{\delta_{m 0}}{2}\right) \frac{1}{2 k_{\rho m}^{2} h} \\
& {\left[(\nabla \times \hat{z})\left(\nabla^{\prime} \times \hat{z}\right) H_{0}^{(2)}\left(k_{\rho m} \mid \rho-\rho^{\prime}\right) f_{c}\left(k_{z m}, z, z^{\prime}\right)\right.} \\
&+\frac{1}{k^{2}}(\nabla \times \nabla \times \hat{z})\left(\nabla^{\prime} \times \nabla^{\prime} \times \hat{z}\right) H_{0}^{(2)} \\
&\left..\left(k_{\rho m} \mid \rho-\rho^{\prime}\right) f_{s}\left(k_{z m}, z, z^{\prime}\right)\right]
\end{aligned}
$$

With $k=\omega \sqrt{\mu \varepsilon_{0} \varepsilon}, k_{z m}=m \pi / h, k_{\rho m}=\sqrt{k^{2}-k_{z m}^{2}}$ and

$\delta_{m 0}=\left\{\begin{array}{lll}1 & \text { for } & m=0 \\ 0 & \text { for } & m \neq 0\end{array}\right.$

$f_{c, s}=\left(\begin{array}{l}\cos \left(k_{z m} z\right) \cos \left(k_{z m} z^{\prime}\right) \\ \sin \left(k_{z m} z\right) \sin \left(k_{z m} z^{\prime}\right)\end{array}\right.$

Where $k$ is the wave number, $k_{z m}$ is the wave number in $z$ direction, and $k_{\rho m}$ is the radial wave number.

\section{REFERENCES}

[1] H.P. Yin \& W.B. Dou, "Analysis of an extended hemi-spherical lens antenna at millimeter wavelengths," Journal of Electromagnetic Waves and Applications, vol.16, No.9, pp.1209-1222, 2002.

[2] G. Godi, R. Sauleau, L. Le Coq, and D. Thouroude, "Design and optimization of three dimensional integrated lens antennas with genetic algorithm," IEEE Transaction on Antennas and Propagation, vol. 55, no. 3, pp. 770-775, Mar. 2007.

[3] L. Mall and R.B. Waterhouse, "Millimeter-wave proximity-coupled microstrip antenna on an extended hemispherical dielectric lens," IEEE Transaction on Antennas and Propagation, vol. 49, No.12, pp. 17691772, 2001.

[4] N.Tinh Nguyen, R. Sauleau, L. Le Coq, "Lens antennas with flat-top radiation patterns: Benchmark of beam shaping techniques at the feed array level and lens shape level," 3rd European Conference on Antennas and Propagation (EuCAP), pp.2834-2837, March 2009.

[5] M. Bozzi, A. Georgiadis and K. Wu, "Review of substrate-integrated waveguide circuits and antennas," IET Microw. Antennas Propag., vol. 5, No. 8, pp. 909-920, 2011.

[6] R. Bayderkhani, K. Forooraghi and B. Abbasi-Arand, "Gain intensified slot antennas backed by SIW cavity using high order cavity resonance," International Journal of Microwave and wireless technologies, accepted for publication, Aug. 2014.

[7] G. Godi, R. Sauleau, D. Thouroude, "Performance of reduced size substrate lens antennas for millimeter-wave communications", IEEE Trans. Antennas Propag., vol. 53, no. 4, pp. 1278-1286, Apr. 2005.

[8] M.J.M. van der Vorst and P.J.I. de Maagt, "Efficient body of revolution finite-difference time-domain modeling of integrated lens antennas," IEEE Microw. Wireless Compon. Lett., vol. 12, no. 7, pp. 258-260, 2002.

[9] M. Kishihara, K. Yamane and I. Ohta, "Analysis of post-wall waveguide by H-plane planar circuit approach," IEEE MTT-S International Microwave Symposium, Honolulu (HA), 2007.

[10] D. Deslandes, L. Perregrini, P. Arcioni, M. Bressan, K. Wu, and G. Conciauro, "Dispersion characteristics of substrate integrated rectangular waveguide," IEEE Microwave Wireless Compon. Lett., vol. 12, pp.333335, Sept. 2002.

[11] E. Abaei, E. Mehrshahi, G. Amendola, E. Arnieri and A. Shamsafar, "Two dimensional multi-port method for analysis of propagation characteristics of substrate integrated waveguide," Progress in Electromagnetics Research C, vol. 29, pp. 261-273, 2012.

[12] E.Arnieri and G. Amendola, "Method of moments analysis of slotted substrate integrated waveguide arrays," IEEE Trans. Antennas and Propag., vol. 59, No.4, pp. 1148-1154, 2011.

[13] E. Arnieri and G. Amendola, "Analysis of substrate integrated waveguide structures based on the parallel-plate waveguide Green's function," IEEE Trans. Microwave Theory and Tech., vol. 56, pp. 1615-1623, 2008.

[14] G. Amendola, E. Arnieri, and L. Boccia, "Analysis of lossy SIW structures based on the parallel plates waveguide green's function," Progress in Electromagnetics Research C, vol. 33, pp. 157-169, 2012. 